\title{
OWL-Eu: Adding customised datatypes into OWL ${ }^{\text {is }}$
}

\author{
Jeff Z. Pan*, Ian Horrocks \\ School of Computer Science, University of Manchester, Kilburn Building, Manchester M13 9PL, UK
}

Received 9 May 2005; received in revised form 20 July 2005; accepted 10 August 2005

\begin{abstract}
Although OWL is rather expressive, it has a very serious limitation on datatypes; i.e., it does not support customised datatypes. It has been pointed out that many potential users will not adopt OWL unless this limitation is overcome, and the W3C Semantic Web Best Practices and Development Working Group has set up a task force to address this issue. This paper makes the following two contributions: (i) it provides a brief summary of OWL-related datatype formalisms, and (ii) it provides a decidable extension of OWL DL, called OWL-Eu, that supports customised datatypes. A detailed proof of the decidability of OWL-Eu is presented.
\end{abstract}

(C) 2005 Elsevier B.V. All rights reserved.

Keywords: Ontologies; Semantic Web; Description Logics; Customised Datatypes; Unary datatype groups

\section{Introduction}

The OWL Web Ontology Language [3] is a W3C recommendation for expressing ontologies in the Semantic Web. Datatype support $[17,18]$ is one of the key features that OWL is expected to provide, and has prompted extensive discussions in the RDFLogic mailing list [21] and in the Semantic Web Best Practices mailing list [23]. Although OWL adds considerable expressive power to the Semantic Web, the OWL datatype formalism (or simply OWL datatyping) is much too weak for many applications; in particular, OWL datatyping does not provide a general framework for customised datatypes, ${ }^{1}$ such as XML Schema derived datatypes.

It has been pointed out that many potential users will not adopt OWL unless this limitation is overcome [22], as it is often necessary to enable users to define their own datatypes and datatype predicates for their ontologies and applications.

Example 1. Customised datatypes are important in capturing the intended meaning of some vocabulary in ontologies. For

\footnotetext{
is This is a revised and extended version of a paper with the same title that was published in the Second European Semantic Web Conference (ESWC2005).

* Corresponding author. Tel.: +44 161275 6139; fax: +44 1612756204

E-mail addresses: pan@cs.man.ac.uk (J. Pan), horrocks@cs.man.ac.uk (I. Horrocks).

${ }^{1}$ A widely discussed example would be the 'BigWheel' example discussed in, e.g., http://lists.w3.org/Archives/Public/public-swbp-wg/2004Apr/0061.html.
}

example, the customised datatype 'atLeast18' can be used in the following definition of the class 'Adult':

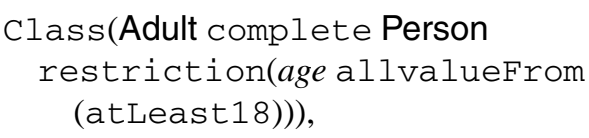

which says that an Adult is a Person whose age is at least 18. The datatype constraint 'at least 18' can be defined as an XML Schema user-defined datatype as follows:

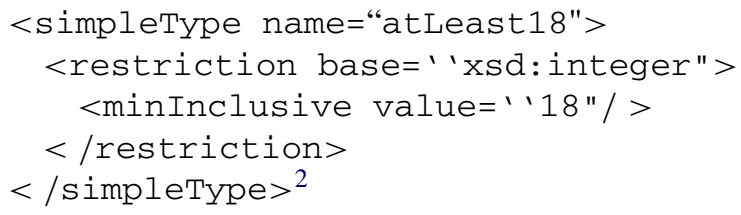

Such user-defined datatypes cannot, however, be used in OWL.

After reviewing the design of OWL, and the needs of various applications and (potential) users, the following requirements for an extension to OWL DL have been identified:

1. It should provide customised datatypes; therefore, it should be based on a datatype formalism which is compatible with OWL datatyping, provides facilities to construct customised

\footnotetext{
${ }^{2}$ More details of XML Schema Datatypes can be found in Section 3.1.
} 
datatypes and, most importantly, guarantees the computability of the kinds of customised datatypes it supports.

2. It should overcome other important limitations of OWL datatyping, such as the absence of negated datatypes and the un-intuitive semantics for unsupported datatypes (which will be further explained in Section 4).

3 . It should satisfy the small extension requirement, which is two folded: on the one hand, the extension should be a substantial and necessary extension that overcomes the above mentioned limitations of OWL datatyping; on the other hand, following W3C's 'one small step at a time' strategy, it should only be as large as is necessary in order to satisfy the requirements.

4. It should be a decidable extension of OWL DL.

This paper makes two main contributions. Firstly, it provides an overview of relevant (to OWL) datatype formalisms, namely those of XML, RDF and OWL itself. Secondly, and most importantly, it presents an extension of OWL DL, ${ }^{3}$ called OWL with unary datatype Expressions (OWL-Eu), which satisfies the above requirements.

The rest of the paper is organised as follows. Section 2 briefly introduces the OWL Web Ontology Language. Section 3 describes OWL-related datatype formalisms. Section 4 summarises the limitations of OWL datatyping. Section 5 presents the OWL-Eu language, showing how it satisfies the above four requirements. Section 6 describes some related work, and Section 7 concludes the paper and suggests some future work.

\section{An overview of OWL}

OWL is a standard (W3C recommendation) for expressing ontologies in the Semantic Web. The OWL language facilitates greater machine understandability of Web resources than that supported by RDFS by providing additional constructors for building class and property descriptions (vocabulary) and new axioms (constraints), along with a formal semantics. The OWL recommendation actually consists of three languages of increasing expressive power: OWL Lite, OWL DL and OWL Full. OWL Lite and $O W L D L$ are, like DAML + OIL, basically very expressive Description Logics (DLs); they are almost ${ }^{4}$ equivalent to the $\mathcal{S H \mathcal { I }} \mathcal{F}\left(\mathbf{D}^{+}\right)$and $\mathcal{S H O I N}\left(\mathbf{D}^{+}\right)$DLs. OWL Full provides the same set of constructors as OWL DL, but allows them to be used in an unconstrained way (in the style of RDF). It is easy to show that OWL Full is undecidable, because it does not impose restrictions on the use of transitive properties [12]; therefore, when we mention OWL in this paper, we usually mean OWL DL.

Let $\mathbf{C}, \mathbf{R}_{\mathbf{I}}, \mathbf{R}_{\mathrm{D}}$ and $\mathbf{I}$ be the sets of URIrefs that can be used to denote concepts, individual-valued properties, data-valued properties and individuals respectively. An OWL DL interpretation is a tuple $\mathcal{I}=\left(\Delta^{\mathcal{I}}, \Delta_{\mathrm{D}},{ }^{\mathcal{I}},{ }^{\mathrm{D}}\right)$ where the individual domain $\Delta^{\mathcal{I}}$ is a nonempty set of individuals, the datatype domain $\Delta_{\mathrm{D}}$ is a nonempty set of data values, ${ }^{\mathcal{I}}$ is an individual interpretation function that maps

\footnotetext{
${ }^{3} \mathrm{cf}$. Section 2 for the differences of three sub-languages of OWL.

${ }^{4}$ They also provide annotation properties, which Description Logics do not.
}

- each individual name $\mathbf{a} \in \mathbf{I}$ to an element $\mathbf{a}^{\mathcal{I}} \in \Delta^{\mathcal{I}}$,

- each concept name $\mathrm{CN} \in \mathbf{C}$ to a subset $\mathrm{CN}^{\mathcal{I}} \subseteq \Delta^{\mathcal{I} \simeq \text {, }}$

- each individual-valued property name $R N \in \mathbf{R}_{\mathbf{I}}$ to a binary relation $R N^{\mathcal{I}} \subseteq \Delta^{\mathcal{I}} \times \Delta^{\mathcal{I}}$ and

- each data-valued property name $T N \in \mathbf{R}_{\mathrm{D}}$ to a binary relation $T N^{\mathcal{I}} \subseteq \Delta^{\mathcal{I}} \times \Delta_{\mathrm{D}}$

and ${ }^{\mathrm{D}}$ is a datatype interpretation function. More details of $\Delta_{\mathrm{D}}$ and ${ }^{\mathrm{D}}$ will be presented in Section 3.3.

Let $R N \in \mathbf{R}_{\mathbf{I}}$ an individual-valued property URIref, $R$ an individual-valued property, $T N \in \mathbf{R}_{\mathrm{D}}$ a data-valued property URIref and $T$ a data-valued property. Valid OWL DL individualvalued properties are defined by the DL syntax:

$R::=R N \mid R^{-}$;

valid OWL DL data-valued properties are defined by the DL syntax:

$T::=T N$.

Let $\mathrm{CN} \in \mathbf{C}$ be a concept name, $C, D$ concept descriptions, $o \in$ I an individual, $u$ an OWL datatype range (cf. Definition 8) and $m \in \mathbb{N}$ an integer. Valid OWL DL concept descriptions are defined by the DL syntax:

$$
\begin{aligned}
C::= & \top|\perp| \mathrm{CN}|\neg C| C \sqcap D|C \sqcup D|\{o\} \\
& \exists R . C|\forall R . C| \geqslant m R, \mid \leqslant m R \\
& \exists T . u|\forall T . u| \geqslant m T, \mid \leqslant m T
\end{aligned}
$$

The individual interpretation function can be extended to give semantics to concept and property descriptions shown in Table 1 , where $\mathrm{A} \in \mathbf{C}$ is a concept URIref, $C, C_{1}, \ldots, C_{n}$ are concept descriptions, $S \in \mathbf{R}_{\mathbf{I}}$ is an individual-valued property URIref, $R$ is an individual-valued property description and $\mathrm{o}, \mathrm{O}_{1}, \mathrm{O}_{2} \in \mathbf{I}$ are individual URIrefs, $u$ is a data range (cf. Definition 8), $T \in$ $\mathbf{R}_{\mathrm{D}}$ is a data-valued property and $\sharp$ denotes cardinality.

An OWL DL ontology can be seen as a DL knowledge base [10], which consists of a set of axioms, including class axioms, property axioms and individual axioms. ${ }^{5}$ Table 2 presents the abstract syntax, DL syntax and semantics of OWL axioms, where $R_{1}, \ldots, R_{n}$ are individual-valued property descriptions. More details of the semantics of OWL DL can be found in [19].

\section{Datatype formalisms}

In this section we will provide a brief overview of the XML, RDF and OWL datatype formalisms.

\subsection{XML Schema Datatypes}

W3C XML Schema Part 2 [4] defines facilities for defining simple types to be used in XML Schema as well as other XML specifications.

Definition 1. An XML Schema simple type $d$ is characterised by a value space, $V(d)$, which is a non-empty set, a lexical space,

\footnotetext{
${ }^{5}$ Individual axioms are also called facts.
} 
Table 1

OWL concept and property descriptions

\begin{tabular}{|c|c|c|}
\hline Abstract syntax & DL syntax & Semantics \\
\hline Class $(\mathrm{A})$ & $A$ & $\mathrm{~A}^{\mathcal{I}} \subseteq \Delta^{\mathcal{I}}$ \\
\hline Class(owl:Thing) & $\top$ & $\top^{\mathcal{I}}=\Delta^{\mathcal{I}}$ \\
\hline Class(owl:Nothing) & $\perp$ & $\perp^{\mathcal{I}}=\emptyset$ \\
\hline intersectionOf $\left(C_{1}, C_{2}, \ldots\right)$ & $C_{1} \sqcap C_{2}$ & $\left(C_{1} \sqcap C_{2}\right)^{\mathcal{I}}=C_{1}^{\mathcal{I}} \cap C_{2}^{\mathcal{I}}$ \\
\hline unionOf $\left(C_{1}, C_{2}, \ldots\right)$ & $C_{1} \sqcup C_{2}$ & $\left(C_{1} \sqcup C_{2}\right)^{\mathcal{I}}=C_{1}^{\mathcal{I}} \cup C_{2}^{\mathcal{I}}$ \\
\hline complementOf $(C)$ & $\neg C$ & $(\neg C)^{\mathcal{I}}=\Delta^{\mathcal{I}} \backslash C^{\mathcal{I}}$ \\
\hline oneOf $\left(\mathrm{o}_{1}, \mathrm{o}_{2}, \ldots\right)$ & $\left\{\mathrm{O}_{1}\right\} \sqcup\left\{\mathrm{O}_{2}\right\}$ & $\left(\left\{\mathrm{o}_{1}\right\} \sqcup\left\{\mathrm{O}_{2}\right\}\right)^{\mathcal{I}}=\left\{\mathrm{o}_{1}^{\mathcal{I}}, \mathrm{o}_{2}^{\mathcal{I}}\right\}$ \\
\hline restriction $(R$ someValuesFrom $(C))$ & $\exists R . C$ & $(\exists R \cdot C)^{\mathcal{I}}=\left\{x \mid \exists y .\langle x, y\rangle \in R^{\mathcal{I}} \wedge y \in C^{\mathcal{I}}\right\}$ \\
\hline restriction $(R$ allValuesFrom $(C))$ & $\forall R . C$ & $(\forall R . C)^{\mathcal{I}}=\left\{x \mid \forall y .\langle x, y\rangle \in R^{\mathcal{I}} \rightarrow y \in C^{\mathcal{I}}\right\}$ \\
\hline restriction $(R$ hasValue(o)) & $\exists R .\{0\}$ & $(\exists R .\{\mathrm{O}\})^{\mathcal{I}}=\left\{x \mid\left\langle x, \mathrm{o}^{\mathcal{I}}\right\rangle \in R^{\mathcal{I}}\right\}$ \\
\hline restriction $(R$ minCardinality $(m))$ & $\geqslant m R$ & $(\geqslant m R)^{\mathcal{I}}=\left\{x \mid \sharp\left\{y .\langle x, y\rangle \in R^{\mathcal{I}}\right\} \geq m\right\}$ \\
\hline $\operatorname{restriction}(R$ maxCardinality $(m))$ & $\leqslant m R$ & $(\leqslant m R)^{\mathcal{I}}=\left\{x \mid \sharp\left\{y \cdot\langle x, y\rangle \in R^{\mathcal{I}}\right\} \leq m\right\}$ \\
\hline restriction $(T$ someValuesFrom $(u))$ & $\exists T . u$ & $(\exists T . u)^{\mathcal{I}}=\left\{x \mid \exists t .\langle x, t\rangle \in T^{\mathcal{I}} \wedge t \in u^{\mathrm{D}}\right\}$ \\
\hline restriction $(T$ allValuesFrom $(u))$ & $\forall T . u$ & $(\forall T . u)^{\mathcal{I}}=\left\{x \mid \exists t .\langle x, t\rangle \in T^{\mathcal{I}} \rightarrow t \in u^{\mathrm{D}}\right\}$ \\
\hline restriction $(T$ hasValue $(w))$ & $\exists T .\{w\}$ & $(\exists T .\{w\})^{\mathcal{I}}=\left\{x \mid\left\langle x, w^{\mathrm{D}}\right\rangle \in T^{\mathcal{I}}\right\}$ \\
\hline restriction $(T$ minCardinality $(m))$ & $\geqslant m T$ & $(\geqslant m T)^{\mathcal{I}}=\left\{x \mid \sharp\left\{t \mid\langle x, t\rangle \in T^{\mathcal{I}}\right\} \geq m\right\}$ \\
\hline restriction $(T$ maxCardinality $(m))$ & $\leqslant m T$ & $(\leqslant m T)^{\mathcal{I}}=\left\{x \mid \sharp\left\{t \mid\langle x, t\rangle \in T^{\mathcal{I}}\right\} \leq m\right\}$ \\
\hline ObjectProperty $(S)$ & $S$ & $S^{\mathcal{I}} \subseteq \Delta^{\mathcal{I}} \times \Delta^{\mathcal{I}}$ \\
\hline ObjectProperty $\left(S^{\prime}\right.$ inverseOf $\left.(S)\right)$ & $S^{-}$ & $\left(S^{-}\right)^{\mathcal{I}} \subseteq \Delta^{\mathcal{I}} \times \Delta^{\mathcal{I}}$ \\
\hline DatatypeProperty $(T)$ & $T$ & $T^{\mathcal{I}} \subseteq \Delta^{\mathcal{I}} \times \Delta_{\mathrm{D}}$ \\
\hline
\end{tabular}

$L(d)$, which is a non-empty set of Unicode [6] strings, and a set of facets, $F(d)$, each of which characterizes a value space along independent axes or dimensions.

XML Schema simple types are divided into disjoint builtin simple types and derived simple types. Derived datatypes can be defined by derivation from primitive or existing derived datatypes by the following three means:
- Derivation by restriction, i.e., by using facets on an existing type, so as to limit the number of possible values of the derived type.

- Derivation by union, i.e., to allow values from a list of simple types.

- Derivation by list, i.e., to define the list type of an existing simple type.

Table 2

OWL axioms

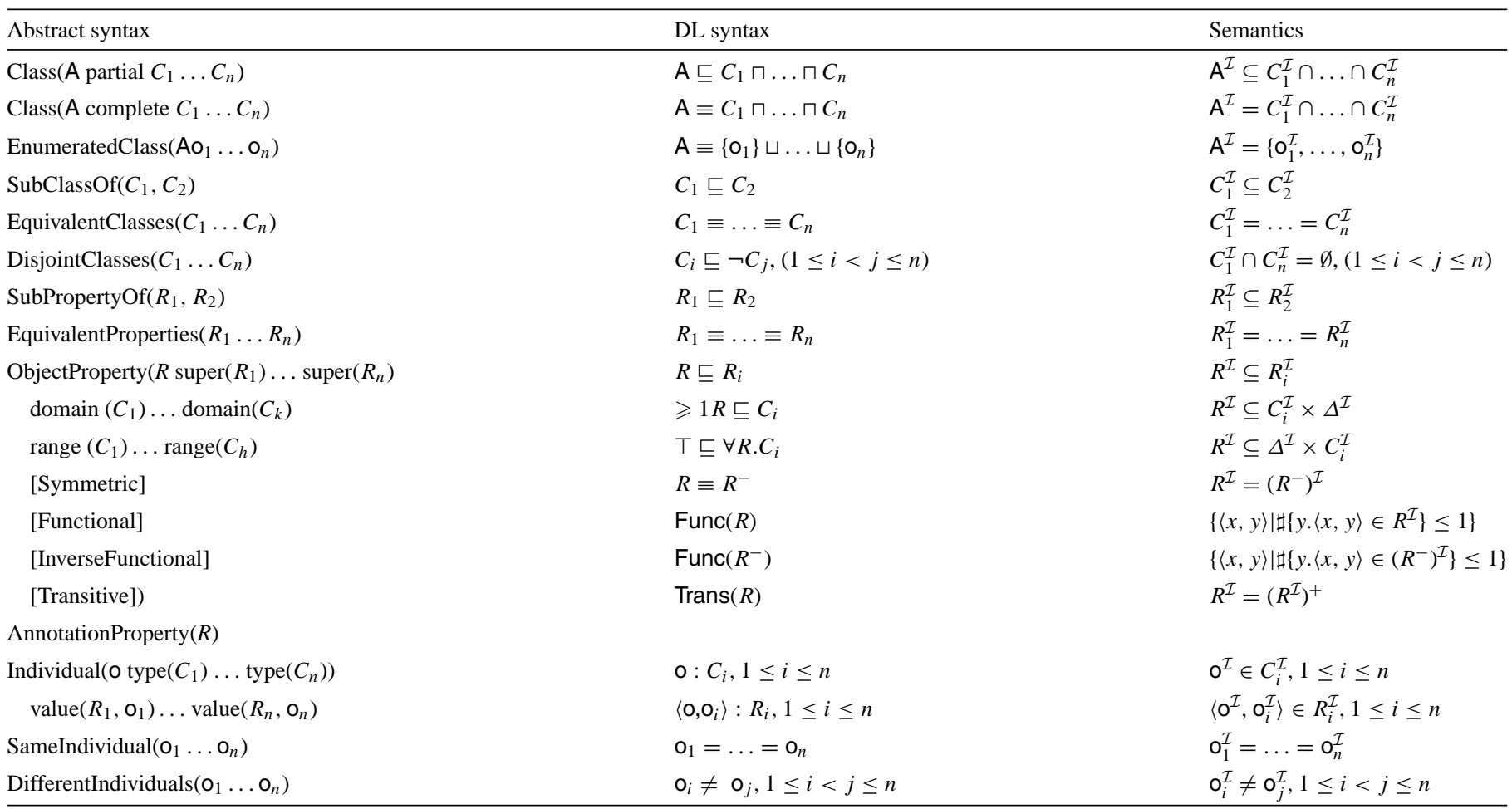


The atLeast 18 datatype defined in Example 1 is a derived simple type (of the base datatype xsd:integer), the value space of which is restricted to integers that are greater than or equal to 18 using the facet minInclusive. The cameraPrice datatype defined in Example 4 is a derived simple type by union.

Details of XML Schema derived simple types by list and complex types can be found in [4]. As they are not consistent with the RDF datatype model to be presented in the next section, they are out of the scope of this paper.

\subsection{Datatypes in $R D F$}

According to [8], RDF allows the use of datatypes defined by any external type systems, e.g., the XML Schema type system, which conform to the following specification.

Definition 2. A datatype $d$ is characterised by a lexical space, $L(d)$, which is an non-empty set of Unicode strings; a value space, $V(d)$, which is an non-empty set, and a total mapping $L 2 V(d)$ from the lexical space to the value space.

This specification allows the use of non-list XML Schema built-in simple types as datatypes in RDF, although some builtin XML Schema datatypes are problematic because they do not fit the RDF datatype model. ${ }^{6}$ Furthermore, comparisons between Definitions 1 and 2 show that RDF does not take XML Schema facets into account, which are essential to define derived simple types.

In RDF, data values are represented by literals.

Definition 3. All literals have a lexical form being a Unicode string. Typed literals are of the form "s" " $u$, where $\mathrm{s}$ is a Unicode string, called the lexical form of the typed literal, and $u$ is a datatype URI reference. Plain literals have a lexical form and optionally a language tag as defined by [1], normalised to lowercase.

Example 2. Boolean is a datatype with value space \{true, false\}, lexical space \{"true", "false", "1", "0"\} and lexical-to-value mapping \{ "true" $\mapsto$ true, "false" $\mapsto$ false, "1" $\mapsto$ true, "0" $\mapsto$ false $\}$. "true"^ Xsd:boolean is a typed literal, while "true" is a plain literal.

The associations between datatype URI references (e.g., xsd:boolean) and datatypes (e.g., boolean) can be provided by datatype maps defined as follows.

Definition 4. A datatype map $\mathbf{M}_{d}$ is a partial mapping from datatype URI references to datatypes.

Note that XML Schema derived simple types are not RDF datatypes because XML Schema provides no mechanism for using URI references to refer to derived simple types.

The semantics of RDF datatypes are defined in terms of $\mathbf{M}_{d^{-}}$ interpretations, which extend RDF-interpretations and RDFSinterpretations (cf. RDF Semantics [8]) with extra conditions for datatypes.

\footnotetext{
${ }^{6}$ Readers are referred to [8] for more details.
}

Definition 5. Given a datatype map $\mathbf{M}_{d}$, an RDFS $\mathbf{M}_{d^{-}}$ interpretation I of a vocabulary $\mathbf{V}$ (a set of URIrefs and plain literals) is any RDFS-interpretation of $\mathbf{V} \cup\left\{u \mid \exists d .\langle u, d\rangle \in \mathbf{M}_{d}\right\}$ which introduces

- a non-empty set IR of resources, called the domain (or universe) of I,

- a set IP (the RDF-interpretation requires IP to be a sub-set of IR) called the set of properties in I,

- a set IC (the RDFS-interpretation requires IC to be a sub-set of IR) called the set of classes in I, and

- a distinguished subset $\mathbf{L V}$ of IR, called the set of literal values, which contains all the plain literals in $\mathbf{V}$,

- a mapping $I S$ from URIrefs in $\mathbf{V}$ to IR,

- a mapping IEXT, called the extension function, from IP to the powerset of IR $\times \mathbf{I R}$,

- a mapping ICEXT, called the class extension function, from IC to the set of subsets of IR,

- a mapping $I L$ from typed literals in $\mathbf{V}$ into IR,

and satisfies the following extra conditions:

1. $\mathbf{L V}=\operatorname{ICEXT}(I S($ rdfs:Literal $))$,

2. for each plain literal $p l, I L(p l)=p l$,

3. for each pair $\langle u, d\rangle \in \mathbf{M}_{d}$,

(a) $\operatorname{ICEXT}(d)=V(d) \subseteq \mathbf{L V}$,

(b) there exist $d \in \mathbf{I R}$ s.t. $I S(u)=d$,

(c) $I S(u) \in I C E X T(I S($ rdfs:Datatype $))$,

(d) for "s"^ $u^{\prime} \in \mathbf{V}, I S\left(u^{\prime}\right)=d$, if $s \in L(d)$, then $I L\left(" s " \wedge u^{\prime}\right)=L 2 V(d)(s), \quad$ otherwise, $\quad \operatorname{IL}\left(\right.$ " s"^^ $\left.u^{\prime}\right) \in$ IR $\backslash \mathbf{L V}$,

4. if $\quad d \in I C E X T(I S$ (rdfs:Datatype)), then $\langle d, I S$ (rdfs: Literal) $) \in I E X T$ (rdfs:subClassOf).

According to Definition 5, $\mathbf{L V}$ is a subset of IR, i.e., literal values are resources. Condition 1 ensures that the class extension of rdfs:Literal is $\mathbf{L V}$. Condition 2 ensures that the plain literals are interpreted as themselves, and that $\mathbf{L V}$ contains interpretations of all valid typed literals of datatypes in $\mathbf{M}_{d}$. Condition $3 \mathrm{a}$ asserts that $\mathrm{RDF}(\mathrm{S})$ datatypes are classes (because datatypes are interpreted using the class extension function ICEXT), Condition $3 \mathrm{~b}$ ensures that there is a resource $d$ for datatype $d$ in $\mathbf{M}_{d}$, and Condition $3 c$ ensures that the class rdfs:Datatype contains the datatypes used in any satisfying $\mathbf{M}_{d}$-interpretation. Condition 3d explains why the range of $I L$ is $\mathbf{I R}$ rather than $\mathbf{L V}$ (because, for "s" ^^ $u$, if $s \notin L(I S(u))$, then $I L(" s " \wedge \wedge u) \notin \mathbf{L V})$; note that this is different from OWL datatypes (cf. Definition 9). Condition 4 requires that $\mathrm{RDF}(\mathrm{S})$ datatypes are sub-classes of rdfs:Literal.

\subsection{Datatypes in $O W L$}

OWL datatyping adopts the RDF specification of datatypes and data values. It extends RDF datatyping by (i) allowing different OWL reasoners to provide different supported datatypes, and (ii) introducing the use of so called enumerated datatypes. 
Definition 6. Given a datatype map $\mathbf{M}_{d}$, a datatype URI reference $u$ is called a supported datatype URI reference w.r.t $\mathbf{M}_{d}$ if there exists a datatype $d$ s.t. $\mathbf{M}_{d}(u)=d$ (in this case, $d$ is called a supported datatype w.r.t. $\mathbf{M}_{d}$ ); otherwise, $u$ is called an unsupported datatype URI reference w.r.t. $\mathbf{M}_{d}$.

Definition 7. Let $y_{1}, \ldots, y_{n}$ be typed literals. An enumerated datatype is of the form oneOf $\left(y_{1}, \ldots, y_{n}\right)$.

The kinds of datatypes provided by OWL are called OWL data ranges, which can be used in datatype-related class descriptions. In fact, in line (13) and (14) of Table 1, $u$ is an OWL data range.

Definition 8. An OWL data range has one of the forms: (i) a datatype URI reference, (ii) an enumerated datatype, or (iii) rdf:Literal.

The semantics of OWL DL datatypes are defined in terms of OWL datatype interpretations.

Definition 9. An OWL datatype interpretation w.r.t. to a datatype map $\mathbf{M}_{d}$ is a pair $\left(\Delta_{\mathrm{D}},{ }^{\mathrm{D}}\right)$, where the datatype domain

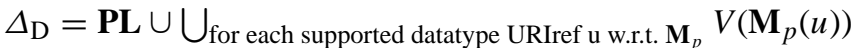
( $\mathbf{P L}$ is the value space for plain literals, i.e., the union of the set of Unicode strings and the set of pairs of Unicode strings and language tags) and ${ }^{D}$ is a datatype interpretation function, which has to satisfy the following conditions:

1. $\operatorname{rdfs}:$ Literal $^{\mathrm{D}}=\Delta_{\mathrm{D}}$

2. for each plain literal $l, l^{\mathrm{D}}=l \in \mathbf{P L}$;

3. for each supported datatype URIref $u\left(\right.$ let $\left.d=M_{d}(u)\right)$ :

(a) $u^{\mathrm{D}}=V(d) \subseteq \Delta_{\mathrm{D}}$

(b) if $s \in L(d)$, then ("s"s $\hat{u} u)^{\mathrm{D}}=L 2 V(d)(s)$,

(c) if $s \notin L(d)$, then ("s" $" \wedge \wedge$ ) $\mathrm{D}$ is not defined;

4. for each unsupported datatype URIref $u, u^{\mathrm{D}} \subseteq \Delta_{\mathrm{D}}$, and (“s”^ $u)^{\mathrm{D}} \in u^{\mathrm{D}}$.

5. each enumerated datatype oneOf $\left(y_{1}, \ldots, y_{n}\right)$ is interpreted as $y_{1}^{\mathrm{D}} \cup \ldots \cup y_{n}^{\mathrm{D}}$.

The above definition shows that OWL datatyping is similar to RDF datatyping, except that (i) RDF datatypes are classes, while OWL DL datatypes are not classes, ${ }^{7}$ and (ii) in RDF ill-defined typed literals are interpreted as resources in $\mathbf{I R} \backslash \mathbf{L V}$, while in OWL DL the interpretation of ill-defined typed literals are undefined.

\section{Limitations of OWL datatyping}

OWL datatyping has the following serious limitations, which discourage potential users from adopting OWL DL in their SW and ontology applications [16,22].

1. OWL does not support customised datatypes (except enumerated datatypes). Firstly, XML Schema derived simple types are not OWL DL datatypes, because of the problem of datatype URI references for XML Schema derived simple types. Secondly, OWL does not provide a mechanism to tell

\footnotetext{
${ }^{7}$ In fact, classes and datatypes in OWL DL use different interpretation functions; cf. Section 2.
}

which (customised) datatypes can be used together so that the language is still decidable.

2. OWL does not support negated datatypes. For example, 'all integers but 0', which is the relativised negation of the enumerated datatype oneOf("0"^ xsd:integer), is not expressible in OWL. Moreover, negated datatypes are necessary in the negated normal form $(\mathrm{NNF})^{8}$ of datatype-related class descriptions in, e.g., DL tableaux algorithms.

3. An OWL DL datatype domain seriously restricts the interpretations of typed literals with unsupported datatype URIrefs. According to Definition 9, datatype domain is equal to the set of all plain literals together with the value spaces of all supported datatypes. For example, given the datatype map $\mathbf{M}_{d 1}=$ xsd:integer $\mapsto$ integer, $x$ sd:string $\mapsto$ string\}, "1.278e- 3 " ^ $\times$ ssd:float has to be interpreted as either an integer, a string or a string with a language tag, which is counter-intuitive.

\section{OWL-Eu}

This section presents OWL-Eu and elaborates how OWL$\mathrm{Eu}$ satisfies the four requirements (listed in Section 1) in the following four sub-sections.

\subsection{Supporting Customised Datatypes}

OWL-Eu supports customised datatypes through unary datatype expressions based on unary datatype groups. Intuitively, an unary datatype group extends the OWL datatyping with a hierarchy of supported datatypes. ${ }^{9}$

Definition 10. A unary datatype group $\mathcal{G}$ is a triple $\left(\mathbf{M}_{d}, \mathbf{B}\right.$, dom), where $\mathbf{M}_{d}$ is the datatype map of $\mathcal{G}, \mathbf{B}$ is the set of primitive base datatype URI references in $\mathcal{G}$ and dom is the declared domain function. We call $\mathbf{S}$ the set of supported datatype URI references of $\mathcal{G}$, i.e., for each $u \in \mathbf{S}, \mathbf{M}_{d}(u)$ is defined; we require $\mathbf{B} \subseteq \mathbf{S}$. We assume that there exists a unary datatype URI reference owlx:DatatypeBottom $\notin \mathbf{S}$. The declared domain function dom has the following properties: for each $u \in \mathbf{S}$, if $u \in \mathbf{B}, \operatorname{dom}(u)=u$; otherwise, $\operatorname{dom}(u)=v$, where $v \in \mathbf{B}$.

Definition 10 ensures that all the primitive base datatype URIrefs of $\mathcal{G}$ are supported $(\mathbf{B} \subseteq \mathbf{S})$ and that each supported datatype URIref relates to a primitive base datatype URIref through the declared domain function dom.

Example 3. $\mathcal{G}_{1}=\left(\mathbf{M}_{d 1}, \mathbf{B}_{1}\right.$, dom $\left._{1}\right)$ is a unary datatype group, where

- $\mathbf{M}_{d 1}=\{$ xsd:integer $\mapsto$ integer, $x$ sd:string $\mapsto$

string, xsd:nonNegativelnteger $\mapsto \geq_{0}$

, xsdx:integerLessThan $\left.\mathrm{N} \mapsto<_{N}\right\}$,

- $\mathbf{B}_{1}=\{x$ sd:string, $x s d:$ integer $\}$, and

\footnotetext{
${ }^{8}$ A concept is in negation normal form iff negation is applied only to atomic concept names, nominals or datatypes.

${ }^{9}$ Note that in [16] datatype groups allow arbitrary datatype predicates, while here we consider only datatypes, which can be regarded as unary datatype predicates.
} 
- dom $_{1}=\{$ xsd:integer $\mapsto$ xsd:integer, xsd:string $\mapsto$ $\mathrm{xsd}$ :string, $\mathrm{xsd}$ :nonNega tivelnteger $\mapsto$

xsd:integer, xsdx:integerLessThanN $\mapsto$ xsd:integer\}.

According to $\mathbf{M}_{d 1}$, we have $\mathbf{S}_{1}=$ \{xsd:integer, xsd:string, xsd:nonNegativelnteger, xsdx:integer

LessThanN\}, hence $\mathbf{B}_{1} \subseteq \mathbf{S}_{1}$. Note that the value space of $<_{N}$ is

$V\left(<_{N}\right)=\{i \in V($ integer $) \mid i<L 2 V($ integer $)(N)\}$,

and by $<_{N}$ we mean there exists a supported datatype $<_{N}$ for each integer $L 2 V($ integer $)(N)$.

Based on a unary datatype group, OWL-Eu provides a formalism (called datatype expressions) for constructing customised datatypes using supported datatypes.

Definition 11. Let $\mathcal{G}$ be a unary datatype group. The set of $\mathcal{G}$-unary datatype expressions in abstract syntax (corresponding DL syntax can be found in Table 3), abbreviated $\operatorname{Dexp} \mathcal{G}$, is inductively defined as follows:

1. atomic expressions $u \in \operatorname{Dexp}(\mathcal{G})$, for a datatype URIref $u$;
2. relativised negated expressions $\operatorname{not}(u) \in \operatorname{Dexp}(\mathcal{G})$, for a datatype URIref $u$;

3. enumerated expressions oneOf $\left(l_{1}, \ldots, l_{n}\right) \in \operatorname{Dexp}(\mathcal{G})$, for literals $l_{1}, \ldots, l_{n}$;

4. conjunctive expressions and $\left(E_{1}, \ldots, E_{n}\right) \in \operatorname{Dexp}(\mathcal{G})$, for datatype expressions $E_{1}, \ldots, E_{n} \in \operatorname{Dexp}(\mathcal{G})$;

5. disjunctive expressions $\operatorname{or}\left(E_{1}, \ldots, E_{n}\right) \in \operatorname{Dexp}(\mathcal{G})$, for datatype expressions $E_{1}, \ldots, E_{n} \in \operatorname{Dexp}(\mathcal{G})$.

Example 4. $\mathcal{G}$-unary datatype expressions can be used to represent XML Schema non-list simple types. Given the unary datatype group $\mathcal{G}_{1}$ presented in Example 3,

built-in XML Schema simple types integer, string, nonNegativeInteger are supported datatypes in $\mathcal{G}_{1}$;

the XML Schema derived simple type (using only one facet) atLeast 18 defined in Example 1 can be represented by the relativised negated expression

not(xsdx:integerLessThan18);

the following XML Schema derived simple type (using more than one facet) humanAge

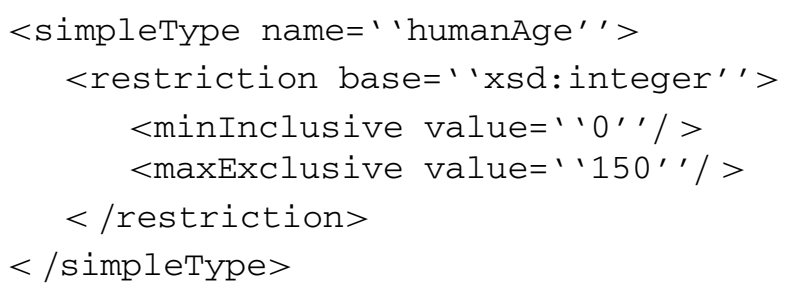

can be represented by the following conjunctive expression

and(xsd:nonNegativelnteger, xsdx:integerLessThan150);

the following XML Schema derived union simple type

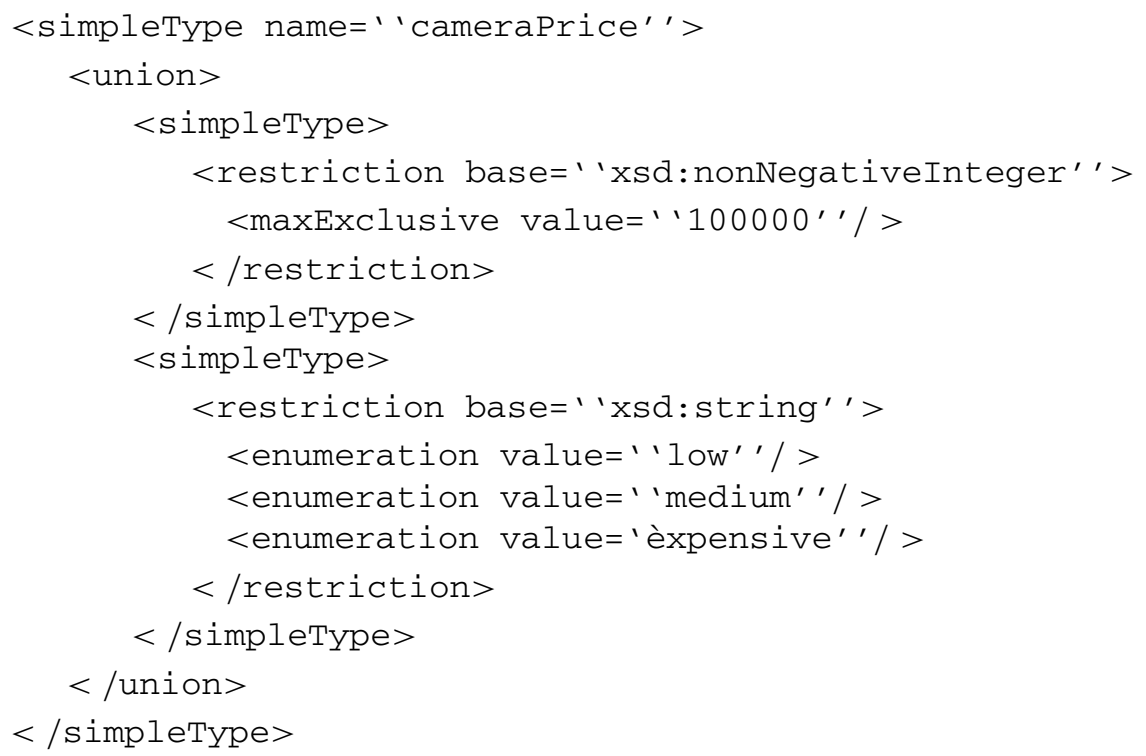


Table 3

Syntax and semantics of datatype expressions (OWL-Eu data ranges)

\begin{tabular}{lll}
\hline Abstract syntax & DL syntax & Semantics \\
\hline a datatype URIref $u$ & $u$ & $u^{\mathrm{D}}$ \\
oneOf $\left(l_{1}, \ldots, l_{n}\right)$ & $\left\{l_{1}, \ldots, l_{n}\right\}$ & $\left\{l_{1}^{\mathrm{D}}\right\} \cup \ldots \cup\left\{l_{n}^{\mathrm{D}}\right\}$ \\
$\operatorname{not}(\mathrm{u})$ & $\bar{u}$ & $(\text { dom }(u))^{\mathrm{D}} \backslash u^{\mathrm{D}}$ if $u \in$ \\
& & $\mathbf{S} \backslash \mathbf{B} \Delta \mathrm{D} \backslash$ \\
$\operatorname{and}\left(E_{1}, \ldots, E_{n}\right)$ & $u^{\mathrm{D}}$ otherwise \\
$\operatorname{or}(P, Q)$ & $E_{1} \wedge \ldots \wedge E_{n}$ & $E_{1}^{\mathrm{D}} \cap \ldots \cap E_{n}^{\mathrm{D}}$ \\
\hline
\end{tabular}

We now define the interpretation of a unary datatype group.

Definition 12. A datatype interpretation $\mathcal{I}_{\mathrm{D}}$ of a unary datatype group $\mathcal{G}=\left(\mathbf{M}_{d}, \mathbf{B}\right.$, dom) is a pair $\left(\Delta_{\mathrm{D}},{ }^{\mathrm{D}}\right)$, where $\Delta_{\mathrm{D}}$ (the datatype domain) is a non-empty set and ${ }^{\mathrm{D}}$ is a datatype interpretation function, which has to satisfy the following conditions:

1. $(\text { rdfs:Literal })^{\mathrm{D}}=\Delta_{\mathrm{D}}$ and $(\text { owlx:DatatypeBottom })^{\mathrm{D}}=\emptyset$;

2. for each plain literal $l, l^{\mathrm{D}}=l \in \mathbf{P L}$ and $\mathbf{P L} \subseteq \Delta_{\mathrm{D}} ;^{10}$

3. for any two primitive base datatype URIrefs $u_{1}, u_{2} \in \mathbf{B}$ $u_{1}^{\mathrm{D}} \cap u_{2}^{\mathrm{D}}=\emptyset$;

4. for each supported datatype URIref $u \in \mathbf{S}$, where $d=$ $\mathbf{M}_{d}(u)$ :

(a) $u^{\mathrm{D}}=V(d) \subseteq \Delta_{\mathrm{D}}, L(u) \subseteq L(\operatorname{dom}(u))$ and $L 2 V(u) \subseteq$ $L 2 V(\operatorname{dom}(u))$

(b) if $s \in L(d)$, then ("s"^^u) $\mathrm{D}=L 2 V(d)(s)$; otherwise, ("s" $\left.{ }^{\wedge} u\right)^{\mathrm{D}}$ is not defined;

5. $\forall u \notin \mathbf{S}, u^{\mathrm{D}} \subseteq \Delta_{\mathrm{D}}$, and "s" $\wedge \wedge \in u^{\mathrm{D}}$.

Moreover, we extend ${ }^{\mathrm{D}}$ to $\mathcal{G}$ unary datatype expression as shown in Table 3. Let $E$ be a $\mathcal{G}$ unary datatype expression, the negation of $E$ is of the form $\neg E$, which is interpreted as $\Delta_{\mathrm{D}} / E^{\mathrm{D}}$.

In Definition 12, Condition 3 ensures that the value spaces of all primitive base datatypes are disjoint with each other. Condition $4 \mathrm{a}$ ensures that each supported datatype is a derived datatype of its primitive base datatype. Please note the difference between a relativised negated expression and the negation of a unary datatype expression: the former one is a kind of unary datatype expression, while the latter one is the form of negation of all kinds of unary datatype expressions. Furthermore, Definition 12 indicates enumerated expressions are special forms of disjunctive expressions.

It is worth noting that the (full) negation of a unary datatype expression is also a unary datatype expression. This can be easily shown as follows.

- $\neg u$ : if $u \in \mathbf{B}, \neg u=\bar{u}$; otherwise, $\neg u=\bar{u} \vee \overline{\operatorname{dom}(u)}$.

- $\neg \bar{u}$ : if $u \in \mathbf{B}, \neg \bar{u}=u$; otherwise, $\neg \bar{u}=u \vee \overline{\operatorname{dom}(u)}$.

- $\neg\left(u_{1} \wedge \ldots \wedge u_{n}\right)=\neg u_{1} \vee \ldots \vee \neg u_{n}$.

- $\neg\left(u_{1} \vee \ldots \vee u_{n}\right)=\neg u_{1} \wedge \ldots \wedge \neg u_{n}$

Next, we introduce the kind of basic reasoning mechanisms required for a unary datatype group.

\footnotetext{
${ }^{10} \mathbf{P L}$ is the value space for plain literals; cf. Definition 9.
}

Definition 13. Let $\mathbf{V}$ be a set of variables, $\mathcal{G}=\left(\mathbf{M}_{d}, \mathbf{B}\right.$, dom $)$ a unary datatype group and $u \in \mathbf{B}$ a primitive base datatype URIref. A datatype conjunction of $u$ is of the form

$\mathcal{C}=\bigwedge_{j=1}^{k} u_{j}\left(v_{j}\right) \wedge \bigwedge_{i=1}^{l} \neq_{i}\left(v_{1}^{(i)}, v_{2}^{(i)}\right)$,

where the $v_{j}$ are variables from $\mathbf{V}, v_{1}^{(i)}, v_{2}^{(i)}$ are variables appear in $\bigwedge_{j=1}^{k} u_{j}\left(v_{j}\right), u_{j}$ are datatype URI references from $\mathbf{S}$ such that $\operatorname{dom}\left(u_{j}\right)=u$, and $\neq_{i}$ are the inequality predicates for primitive base datatypes $\mathbf{M}_{d}\left(\operatorname{dom}\left(u_{i}\right)\right)$ where $u_{i}$ appear in $\bigwedge_{j=1}^{k} u_{j}\left(v_{j}\right)$.

A datatype conjunction $\mathcal{C}$ is called satisfiable iff there exist an interpretation $\left(\Delta_{\mathrm{D}},{ }^{\mathrm{D}}\right)$ of $\mathcal{G}$ and a function $\delta$ mapping the variables in $\mathcal{C}$ to data values in $\Delta_{\mathrm{D}}$ s.t. $\delta\left(v_{j}\right) \in u_{j}^{\mathrm{D}}$ (for all $1 \leq$ $j \leq k)$ and $\left\{\delta\left(v_{1}^{(i)}\right), \delta\left(v_{2}^{(i)}\right)\right\} \subseteq u_{i}^{\mathrm{D}}$ and $\delta\left(v_{1}^{(i)}\right) \neq \delta\left(v_{2}^{(i)}\right)$ (for all $1 \leq$ $i \leq l)$. Such a function $\delta$ is called a solution for $\mathcal{C}$ w.r.t. $\left(\Delta_{\mathrm{D}},{ }^{\mathrm{D}}\right)$.

We end this section by elaborating the conditions that computable unary datatype groups require.

Definition 14. A unary datatype group $\mathcal{G}$ is conforming iff

1. for any $u \in \mathbf{S} \backslash \mathbf{B}$ : there exist $u^{\prime} \in \mathbf{S} \backslash \mathbf{B}$ such that $u^{\prime \mathrm{D}}=\bar{u}^{\mathrm{D}}$, and

2. for each primitive base datatype in $\mathcal{G}$, the satisfiability problems for finite datatype conjunctions of the form (1) is decidable.

\subsection{Small extension: from $O W L D L$ to $O W L-E u$}

In this section, we present a small extension of OWL DL, i.e.,

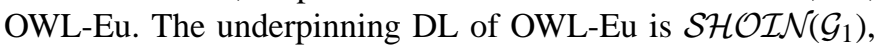
i.e., the $\mathcal{S H O} \mathcal{I N}$ DL combined with a unary datatype group $\mathcal{G}$ (1 for unary). Specifically, OWL-Eu (only) extends OWL data range (cf. Definition 8) to OWL-Eu data ranges defined as follows.

Definition 15. An $O W L-E u$ data range is a $\mathcal{G}$ unary datatype expression. Abstract (as well as DL) syntax and model-theoretic semantics of OWL-Eu data ranges are presented in Table 3.

The consequence of the extension is that customised datatypes, represented by OWL-Eu data ranges, can be used in datatype exists restrictions $(\exists T . u)$ and datatype value restrictions $(\forall T . u)$, where $T$ is a datatype property and $u$ is an OWL-Eu data range (cf. Table 1). Hence, this extension of OWL DL is as large as is necessary to support customised datatypes.

Example 5. PCs with memory size greater than or equal to 512 $\mathrm{Mb}$ and with price cheaper than 700 pounds can be represented in the following OWL-Eu concept description in DL syntax (cf. Table 3):

\section{$\mathrm{PC} \sqcap \exists$ memorySizeIn $M b . \overline{<512} \sqcap$}

$\exists$ priceIn Pound. $<700$,

where $\overline{<512}$ is a relativised negated expression and $<_{700}$ is a supported datatype in $\mathcal{G}_{1}$. 


\subsection{Decidability of $O W L-E u$}

Now we show that OWL-Eu is decidable by showing $\mathcal{S H O} \mathcal{I} \mathcal{Q}\left(\mathcal{G}_{1}\right)$-concept satisfiability w.r.t. knowledge bases. To decide $\mathcal{S H O I} \mathcal{O}\left(\mathcal{G}_{1}\right)$-concept satisfiability and subsumption problem w.r.t. knowledge bases, a DL reasoner can use a datatype reasoner to answer datatype queries. Intuitively, a datatype query is a disjunction of datatype expression conjunctions, possibly together with some equality and inequality constraints.

Definition 16. (Datatype Query) For a unary datatype group $\mathcal{G}_{1}$, a datatype query is of the form

$$
\begin{aligned}
\mathcal{Q} & :=\bigvee_{j=1}^{k} \mathcal{C}_{d_{j}} \wedge \bigwedge_{j_{1}=1}^{k_{1}} \neq\left(v_{\left(j_{1}, 1\right)}, v_{\left(j_{1}, 2\right)}\right) \wedge \bigvee_{j_{2}=1}^{k_{2}} \\
& =\left(v_{\left(j_{2}, 1\right)}, \ldots, v_{\left(j_{2}, m_{j_{2}}\right)}\right),
\end{aligned}
$$

where $\mathcal{C}_{d_{j}}$ is a (possibly negated) unary datatype expression conjunction, $v_{(s)}$ are variables appearing in $\mathcal{C}_{d_{1}}, \ldots, \mathcal{C}_{d_{k}}$, and $\neq$ and $=$ are called the value inequality predicate and value equality predicate, respectively. A datatype query is satisfiable iff there exists an interpretation $\left(\Delta_{\mathrm{D}},{ }^{\mathrm{D}}\right)$ of $\mathcal{G}_{1}$ and a function $\delta$ mapping the variables in $\mathcal{C}_{d_{1}}, \ldots, \mathcal{C}_{d_{k}}$ to data values in $\Delta_{\mathrm{D}}$ s.t.

$\delta$ is a solution for one of $\mathcal{C}_{d_{1}}, \ldots, \mathcal{C}_{d_{k}}$ w.r.t. $\left(\Delta_{\mathrm{D}},{ }^{\mathrm{D}}\right)$ and, $\delta\left(v_{\left(j_{1}, 1\right)}\right) \neq \delta\left(v_{\left(j_{1}, 2\right)}\right)$ for all $1 \leq j_{1} \leq k_{1},{ }^{11}$

there exist some $j_{2}\left(1 \leq j_{2} \leq k_{2}\right)$ s.t. $\delta\left(v_{\left(j_{2}, 1\right)}\right)=\cdots=$ $\delta\left(v_{\left(j_{2}, m_{j_{2}}\right)}\right)$.

Such a function $\delta$ is called a solution for $\mathcal{Q}$ w.r.t. $\left(\Delta_{\mathrm{D}},{ }^{\mathrm{D}}\right)$.

Lemma 1. For $\mathcal{G}$ a conforming unary datatype group, datatype queries of the form (2) are decidable.

Proof. Firstly, we will show that the satisfiability problem of (possibly negated) $\mathcal{G}$-datatype expression conjunctions is decidable. It is trivial to reduce the satisfiability problem for $\mathcal{G}$ datatype expression conjunctions to the satisfiability problem for predicate conjunctions over $\mathcal{G}$ :

1. Due to Condition 1 of a conforming unary datatype group (cf. Definition 14), we can trivially eliminate relativised negated expressions. Similarly, their (full) negations can be reduced as follows:

$\neg \overline{u_{i}}\left(v_{i}\right) \equiv \begin{cases}u_{i}\left(v_{i}\right) \vee \overline{\operatorname{dom}}\left(u_{i}\right)\left(v_{i}\right) & \text { if } u_{i} \in \mathbf{S} \backslash \mathbf{B}, \\ u_{i}\left(v_{i}\right) & \text { otherwise, }\end{cases}$

according to Definition 12.

2. The and and or constructors simply introduce disjunctions of datatype conjunctions of $\mathcal{G}$. Due to Condition 3 of Definition 12, datatype conjunctions are unsatisfiable if there exist variables shared among supported datatypes derived from different primitive based datatypes. Therefore, datatype conjunctions of $\mathcal{G}$ can be reduced to datatype conjunctions of primitive base datatypes. According to Definition 14,

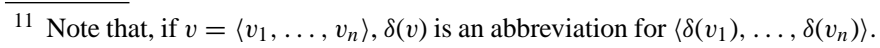

the satisfiability problem of datatype conjunctions of primitive base datatypes is decidable; therefore, a $\mathcal{G}$-datatype expression conjunction is satisfiable iff one of its disjuncts is satisfiable.

Secondly, we show how to handle the extra constraints introduced by the value inequality predicate and value equality predicate. We can transform the general equality and inequality constraints into $\mathcal{V}$, a disjunction of conjunctions of the forms $=\left(v_{i}, v_{j}\right)$ or $\neq\left(v_{i^{\prime}}, v_{j^{\prime}}\right)$. For each satisfiable $\mathcal{G}$-datatype expression conjunction $\mathcal{C}_{E_{j}}$, we can further extend $\mathcal{C}_{E_{j}}$ to $\mathcal{C}_{E_{j}}^{\prime}$ by adding new conjuncts $=_{u}\left(v_{i}, v_{j}\right)$ and/or $\neq_{u}\left(v_{i^{\prime}}, v_{j^{\prime}}\right)$ into $\mathcal{C}_{E}$. $\mathcal{Q}$ is unsatisfiable if all $\mathcal{C}_{E_{j}}^{\prime}$ are unsatisfiable; otherwise, $\mathcal{Q}$ is satisfiable.

We will show the decidability of $\mathcal{S H O I} \mathcal{H}\left(\mathcal{G}_{1}\right)$-concept satisfiability w.r.t. TBoxes and RBoxes by reducing it to the $\mathcal{S H O I Q -}$ concept satisfiability w.r.t. TBoxes and RBoxes. The proof is inspired by the proof (Lutz [14, pp. 32-33]) of the decidability of $\mathcal{A L C F}(\mathcal{D})$-concept satisfiability w.r.t. to general TBoxes, where $\mathcal{A L C} \mathcal{F}(\mathcal{D})$ is obtained from $\mathcal{A} \mathcal{L C} \mathcal{F}(\mathcal{D})$ by restricting the concrete domain constructor to concrete features in place of feature chains. The basic idea behind the reduction is that we can replace each datatype group-based concept $C$ in $\mathcal{T}$ with a new atomic primitive concept $A_{C}$ in $\mathcal{T}^{\prime}$. We then compute the satisfiability problem for all possible conjunctions of datatype groupbased concepts (and their negations) in $\mathcal{T}$ (of which there are only a finite number), and in case a conjunction $C_{1} \sqcap \ldots \sqcap C_{n}$ is unsatisfiable, we add an axiom $A_{C_{1}} \sqcap \ldots \sqcap A_{C_{n}} \sqsubseteq \perp$ to $\mathcal{T}^{\prime}$.

For example, unary datatype group-based concepts $\exists T$. $_{>}$ and $\forall T . \leq_{0}$ occurring in $\mathcal{T}$ would be replaced with $A_{\exists T .>_{1}}$ and $A_{\forall T: \leq_{0}}$ in $\mathcal{T}^{\prime}$, and $A_{\exists T .>1} \sqcap A_{\forall T: \leq_{0}} \sqsubseteq \perp$ would be added to $\mathcal{T}^{\prime}$ because $\exists T .>_{1} \sqcap \forall T . \leq_{0}$ is unsatisfiable (i.e., there is no solution for the predicate conjunction $>_{1}(v) \wedge \leq_{0}(v)$ ).

Theorem 1. The $\mathcal{S H O I} \mathcal{O}\left(\mathcal{G}_{1}\right)$-concept satisfiability problem w.r.t. a knowledge base is decidable if the combined unary datatype group is conforming.

Proof. We prove the theorem by reducing $\mathcal{S H O I}\left(\mathcal{G}_{1}\right)$ concept satisfiability w.r.t. a knowledge base to the $\mathcal{S H O I Q}$ concept satisfiability w.r.t. TBoxes and RBoxes. Let $D$ be an $\mathcal{S H O I} \mathcal{Q}\left(\mathcal{G}_{1}\right)$-concept for satisfiability checking, $\mathrm{cl}_{\mathcal{T}}(D)$ the set of all the sub-concepts of concepts in $\{D\} \cup\left\{D_{1}, D_{2} \mid D_{1} \sqsubseteq\right.$ $D_{2} \in \mathcal{T}_{\text {or }} D_{1}=D_{2} \in \mathcal{T}$, and $\left\{C_{1}, \ldots, C_{k}\right\} \subseteq \operatorname{cl}_{\mathcal{T}}(D)$ the set of all the datatype group-based concepts (and their negations) in $\mathrm{cl}_{\mathcal{T}}(D)$, i.e., each $C_{i}(1 \leq i \leq k)$ is of one of the four forms: $\exists T . d, \forall T . d, \leqslant n T . d$ and $\geqslant n T . d$, where $T$ is a concrete role name, $d$ is a unary datatype expression and $n$ is an integer. There are two remarks here. Firstly, we assume that $C_{1}, \ldots, C_{k}$ are in their negation normal forms; i.e., negations only appear in front of atomic concepts. Secondly, as we have shown in Section 5.1, negations of unary datatype expressions are still unary datatype expressions.

We assume that all the functional concrete role axioms in $\mathcal{R}$ of the form $\operatorname{Func}(T)$ are encoded into concept inclusion axioms of the form $T \sqsubseteq \leqslant 1 T . \top_{\mathrm{D}}$ in $\mathcal{T}$. We assume that all the individual axioms of the form a : $C$ are encoded into concept inclusion axioms of the form $\{\mathrm{a}\} \sqsubseteq C$, that all the individual axioms of 
the form $\langle\mathrm{a}, \mathrm{b}\rangle: R$ are encoded into concept inclusion axioms of the form $\{\mathrm{a}\} \sqsubseteq \exists R .\{\mathrm{b}\}$ and that all the individual axioms of the form $\langle\mathrm{a}, l\rangle: T$ are encoded into concept inclusion axioms of the form $\{\mathrm{a}\} \sqsubseteq \exists T .\{l\}$.

We define a mapping $\pi$ that maps unary datatype group-based concept conjunctions of the form $S=B_{1} \sqcap \ldots \sqcap B_{h}$, where $\left\{B_{1}, \ldots B_{h}\right\} \subseteq\left\{C_{1}, \ldots, C_{k}\right\}$, to a corresponding datatype query $\pi(S)$.

(Step 1) For each $B_{j}$ of the form $\exists T . d, \pi(S)$ contains a conjunct $d\left(v_{j}^{T}\right)$, where each $v_{j}^{T}$ is a variable, with the corresponding concrete role name $T$ as its superscript.

(Step 2) For each $B_{j}$ of the form $\geqslant n T . d, \pi(S)$ contains a conjunct

$$
\bigwedge_{a=1}^{m} d\left(v_{j_{a}}^{T}\right) \wedge \bigwedge_{1 \leq a<b \leq m} \neq\left(v_{j_{a}}^{T}, v_{j_{b}}^{T}\right)
$$

where the inequality constraints are used to make sure the variables $v_{j_{1}}^{T} \ldots, v_{j_{m}}^{T}$ are mapped to different data values. We will not introduce any more new variables (with superscriptions) in the following steps.

(Step 3) For each $B_{j}$ of the form $\forall T . d$, let $A_{j}$ be the set of all variables that were introduced in (Step 1) and (Step 2) of the form $v^{T^{\prime}}$, where the superscript $T^{\prime}$ matches the corresponding concrete role name $T$ in $\forall T$.d. A variable $v^{T^{\prime}}$ matches a concrete role $T$ if $T^{\prime} \stackrel{\Xi}{=} T$. Then $\pi(S)$ contains a conjunct

$$
\bigwedge_{\forall v \in A_{j}} d(v)
$$

(Step 4) For each $B_{j}$ of the form $\leqslant m T_{1}, \ldots, T_{n_{j}}$. E, similarly to (Step 3), we can define a set $A_{j}$ for $B_{j}$. Let $\left|A_{j}\right|=m^{\prime}$. If $m^{\prime} \leq m$, then let $P(A, x)$ be the function that maps a set $A$ to the set of all the partitions of $A$ with size $x$; i.e., for each partition $Q=\left\{q_{1}, \ldots, q_{x}\right\} \in P(A, x), q_{1}, \ldots, q_{n}$ are non-empty sets, $q_{a} \cap q_{b}=\emptyset$ (for $1 \leq a<b \leq x$ ) and $A=q_{1} \cup \ldots \cup q_{x}$. Then $\pi(S)$ contains a conjunct

$$
\bigvee_{Q \in P\left(A_{j}, m\right)} \bigwedge_{q \in Q} \bigwedge_{v_{1}, v_{2} \in q} d\left(v_{1}\right) \wedge d\left(v_{2}\right) \rightarrow=\left(v_{1}, v_{2}\right),
$$

we can apply the " $x \Rightarrow y \equiv \neg x \vee y$ " equivalence and DeMorgan's law to this conjunct to give

$$
\bigvee_{Q \in P\left(A_{j}, m\right)} \bigwedge_{q \in Q} \bigwedge_{v_{1}, v_{2} \in q} \neg d\left(v_{1}\right) \vee \neg d\left(v_{2}\right) \vee=\left(v_{1}, v_{2}\right) \text {. }
$$

Since the satisfiability problem for a datatype query is decidable, for each possible $S=B_{1} \sqcap \ldots \sqcap B_{h}$, where $\left\{B_{1}, \ldots, B_{h}\right\} \subseteq\left\{C_{1}, \ldots, C_{k}\right\}$, we can decide if $\pi(S)$ is satisfiable or not.

Now we can reduce the $\mathcal{S H O I Q}\left(\mathcal{G}_{1}\right)$-concept satisfiability problem w.r.t. a knowledge base to the $\mathcal{S H O I Q}$-concept satisfiability problem w.r.t. a TBox and an RBox, by introducing some new atomic primitive concepts (to represent $C_{i}$, for each $1 \leq i \leq k$ ) and some concept inclusion axioms about these atomic primitive concepts (to capture all the possible contradictions caused by $S$ ) as follows:
(1) We create an atomic primitive concept $A_{C_{i}}$ for each $C_{i} \in$ $\left\{C_{1}, \ldots, C_{k}\right\}$, and transform $\mathcal{T}$ and $D$ into $\mathcal{T}^{\prime}$ and $D^{\prime}$ by replacing all $C_{i}$ with $A_{C_{i}}$ in $\mathcal{T}$ and $D$. We transform $\mathcal{R}$ into $\mathcal{R}^{\prime}$ by removing all the concrete role inclusion axioms.

(2) For each $S=B_{1} \sqcap \ldots \sqcap B_{h}$, where $\left\{B_{1}, \ldots, B_{h}\right\} \subseteq$ $\left\{C_{1}, \ldots, C_{k}\right\}$, if $\pi(S)$ is unsatisfiable, we add the following concept inclusion axiom into $\mathcal{T}^{\prime}$ :

$$
A_{B_{1}} \sqcap \ldots \sqcap A_{B_{h}} \sqsubseteq \perp \text {. }
$$

Claim. (i) For any $S=B_{1} \sqcap \ldots \sqcap B_{h}$, where $\left\{B_{1}, \ldots, B_{h}\right\} \subseteq$ $\left\{C_{1}, \ldots, C_{k}\right\}, S$ is satisfiable iff $\pi(S)$ is satisfiable. (ii) All the possible contradictions caused by possible datatype groupbased sub-concept conjunctions in $\mathrm{cl}_{\mathcal{T}}(D)$ have been encoded in the TBox $\mathcal{T}^{\prime}$. (iii) $D$ is satisfiable w.r.t. $\mathcal{T}$ and $\mathcal{R}$ iff $D^{\prime}$ is satisfiable w.r.t. $\mathcal{T}^{\prime}$ and $\mathcal{R}^{\prime}$.

Claim (i) is true because the mappings in (Steps 1-4) exactly generate the needed datatype queries $\pi(S)$ according to the semantics of unary datatype group-based concepts.

- (Step 1): For each $B_{j}$ of the form $\exists T . d, \pi(S)$ contains a conjunct $d\left(v_{j}^{T}\right)$. If $\left(\Delta_{\mathrm{D}},{ }^{\mathrm{D}}\right)$ is an interpretation of $\mathcal{G}$ and $\delta$ is a solution of $d\left(v_{j}^{T}\right)$ w.r.t. $\left(\Delta_{\mathrm{D}},{ }^{\mathrm{D}}\right)$ of this conjunct, we have $\delta\left(v_{j}^{T}\right) \in d^{\mathrm{D}}$. Furthermore, the concrete role names $T$ are used in superscripts of the corresponding variables, so as to assure that further constraints from datatype expression value and atmost restrictions can be properly added to these variables.

- (Step 2): For each $B_{j}$ of the form $\geqslant m T . d, \pi(S)$ contains a conjunct $\bigwedge_{a=1}^{m} d\left(v_{j_{a}}^{T}\right) \wedge \bigwedge_{1 \leq a<b \leq m} \neq\left(v_{j_{a}}^{T}, v_{j_{b}}^{T}\right)$. If $\left(\Delta_{\mathrm{D}},{ }^{\mathrm{D}}\right)$ is an interpretation of $\mathcal{G}$ and $\delta$ is a solution w.r.t. $\left(\Delta_{\mathrm{D}},{ }^{\mathrm{D}}\right)$ of this conjunct, we have $\delta\left(v_{j_{a}}^{T}\right) \in d^{\mathrm{D}}$ and $\delta\left(v_{j_{a}}^{T}\right) \neq \delta\left(v_{j_{b}}^{T}\right)$ for all $1 \leq a<b \leq m$; viz. there are at least $m$ data values that satisfy the unary datatype expression $d$. The purpose of using superscripts in variables is the same as (Step 1).

- (Step 3): For each $B_{j}$ of the form $\forall T . d, \pi(S)$ contains a conjunct $\bigwedge_{v \in A_{j}} d(v)$. Since in (Step 1) and (Step 2) we have generated all the needed variables, the set $A_{j}$ includes all the tuples of variables, the superscripts of which match $T_{1}, \ldots, T_{n_{j}}$. If $\left(\Delta_{\mathrm{D}},{ }^{\mathrm{D}}\right)$ is an interpretation of $\mathcal{G}$ and $\delta$ is a solution w.r.t. $\left(\Delta_{\mathrm{D}},{ }^{\mathrm{D}}\right)$ of the above conjunct, we have $\delta(v) \in d^{\mathrm{D}}$, for all $v \in A_{j}$.

- (Step 4): For each $B_{j}$ of the form $\leqslant m T . d, \pi(S)$ contains a conjunct

$$
\bigvee_{Q \in P\left(A_{j}, m\right)} \bigwedge_{q \in Q \rightarrow v_{1}, \rightarrow v_{2} \in q} \bigwedge_{q} d\left(v_{1}\right) \wedge d\left(v_{2}\right) \rightarrow=\left(v_{1}, v_{2}\right)
$$

if $m<\left|A_{j}\right|$. The set $A_{j}$ is constructed as that in (Step 3), and $P\left(A_{j}, m\right)$ is the set of all the partitions of $A_{j}$ with size $m$. If $\left(\Delta_{\mathrm{D}},{ }^{\mathrm{D}}\right)$ is an interpretation of $\mathcal{G}$ and $\delta$ is a solution w.r.t. $\left(\Delta_{\mathrm{D}},{ }^{\mathrm{D}}\right)$ of this conjunct, there exists a partition $Q$, s.t. for all $q_{i} \in Q(1 \leq i \leq m)$, any pairs of variable $v_{1}, v_{2}$ must satisfy that if both $\delta\left(v_{1}\right) \in d^{\mathrm{D}}$ and $\delta\left(v_{2}\right) \in d^{\mathrm{D}}$ are true, then $\delta\left(v_{1}\right)=\delta\left(v_{2}\right)$. In other words, there are at most $m$ different data values that are linked through the concrete roles $T$ and satisfy $d$. 
For claim (ii). Firstly, due to the (1), it is obvious that $D^{\prime}$ is

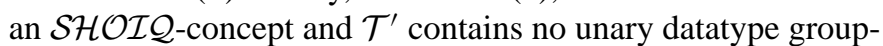
based concepts, and there are no concrete roles in $\mathcal{R}^{\prime}$. Secondly, due to (2), claim (i) and that $\mathcal{G}$-datatype queries are decidable, for any possible datatype group-based concept conjunction $S=B_{1} \sqcap \ldots \sqcap B_{h}$ and if $\pi(S)$ is unsatisfiable, there is an axiom $A_{B_{1}} \sqcap \ldots \sqcap A_{B_{h}} \sqsubseteq \perp$ in $\mathcal{T}^{\prime}$. Therefore, all the possible contradictions caused by possible datatype group-based sub-concept conjunctions in $\mathrm{Cl}_{\mathcal{T}}(D)$ have been encoded in the TBox $\mathcal{T}^{\prime}$.

For claim (iii). If $D$ is satisfiable w.r.t. $\mathcal{T}$ and $\mathcal{R}$, then there is a model $\mathcal{I}$, s.t. $\mathcal{I} \models D, \mathcal{I} \models \mathcal{T}$ and $\mathcal{I} \models \mathcal{R}$. We show how to construct a model $\mathcal{I}^{\prime}$ of $D^{\prime}$ w.r.t. $\mathcal{T}^{\prime}$ and $\mathcal{R}^{\prime}$ from $\mathcal{I}$. $\mathcal{I}^{\prime}$ will be identical to $\mathcal{I}$ in every respect except for concrete roles (there are no concrete roles in $\mathcal{I}^{\prime}$ ) and the atomic primitive concepts $A_{C_{i}}$ for each $C_{i} \in\left\{C_{1}, \ldots, C_{k}\right\}$ (there are no $A_{C_{i}}$ in $\mathcal{I}$ ). So we only need to construct $A_{C_{i}}^{\mathcal{I}^{\prime}}: A_{C_{i}}^{\mathcal{I}^{\prime}}=C_{i}^{\mathcal{I}}$. Due to the constructions of $D^{\prime}, \mathcal{T}^{\prime}, \mathcal{R}^{\prime}$, we have $D^{\prime \mathcal{I}^{\prime}} \neq \emptyset, \mathcal{I}^{\prime} \models \mathcal{T}^{\prime}$ and $\mathcal{I}^{\prime} \models \mathcal{R}^{\prime}$.

For the converse direction, let $\mathcal{I}^{\prime}$ be a model of $D^{\prime}$ w.r.t. $\mathcal{T}^{\prime}$ and $\mathcal{R}^{\prime}$. $\mathcal{I}$ will be identical to $\mathcal{I}^{\prime}$ in every respect except for concrete roles and datatype group-based concepts $C_{1}, \ldots, C_{k}$. We can construct $C_{i}^{\mathcal{I}}(1 \leq i \leq k)$ as $C_{i}^{\mathcal{I}}=A_{C_{i}}^{\mathcal{I}}$ and the interpretations of concrete roles as follows: Let $C=C_{1}^{\mathcal{I}} \cup \ldots \cup$ $C_{k}^{\mathcal{I}}$. For each $x_{j} \in C$, there exists a set $\left\{C_{j_{1}}, \ldots, C_{j_{n_{x}}}\right\}$ s.t. for each $C_{j_{h}} \in\left\{C_{j_{1}}, \ldots, C_{j_{n_{x}}}\right\}, x_{j} \in C_{j_{h}}^{\mathcal{I}}$. Let $S_{j}=C_{j_{1}} \sqcap \ldots \sqcap$ $C_{j_{n_{x}}}$. Obviously, $\mathcal{I} \models S_{j}$. Due to claim (i), the datatype query $\pi\left(S_{j}\right)$ is decidable; therefore, there exists a datatype interpretation $\left(\Delta_{\mathrm{D}},{ }^{\mathrm{D}}\right)$ and a solution $\delta$ of $\pi\left(S_{j}\right)$ w.r.t. $\left(\Delta_{\mathrm{D}},{ }^{\mathrm{D}}\right)$. Let $T$ be a concrete role, $V_{T}^{(j)}$ the set of variables in $\pi\left(S_{j}\right)$ that match $T, \delta\left(V_{T}^{(j)}\right)$ the set of data values to which $\delta$ maps the set of variables in $V_{T}^{(j)}$. Initially, we set all $T^{\mathcal{I}}$ as $\emptyset$, then for each $T$ used in each $S_{j}$, we have $T^{\mathcal{I}}=T^{\mathcal{I}} \cup\left\{S_{j}^{\mathcal{I}} \times \delta\left(V_{T}^{(j)}\right)\right\}$. Obviously, we have $\mathcal{I} \models D$. Due to claim (ii) and the construction of $\mathcal{T}^{\prime}$, we have $\mathcal{I} \models \mathcal{T}$. Due to the definition of match, the constructions of $\mathcal{R}^{\prime}$ and the interpretations of concrete roles, we have $\mathcal{I} \models \mathcal{R}$.

Since OWL-Eu corresponds to the $\mathcal{S H O I N}\left(\mathcal{G}_{1}\right)$ DL, which is a sub-language of $\mathcal{S H O I Q}\left(\mathcal{G}_{1}\right)$, we have the following corollary.

Corollary 1. The OWL-Eu-concept satisfiability problem w.r.t. a knowledge base is decidable.

Lemma 2. (Tobies[24, Lemma 5.3]) If $\mathcal{L}$ is a $D L$ that provides the nominal constructor, knowledge base satisfiability can be polynomially reduced to satisfiability of TBoxes and RBoxes.

According to Corollary 1 and Lemma 5.3, we have the following theorem.

Theorem 2. The knowledge base satisfiability problem of $O W L$ Eu is decidable.

\subsection{Overcoming the limitations of OWL datatyping}

This section summarises how OWL-Eu overcomes the limitations of OWL datatyping presented in Section 4. Firstly, OWL-Eu is a decidable extension (Theorem 1) of OWL DL that supports customised datatypes with unary datatype expressions (cf. Example 4). Secondly, Definition 12 defines the negations of datatype expressions and OWL-Eu provides relativised negated datatype expression (Definition 11). Thirdly, according to Definition 12, the datatype domain in an interpretation of a datatype group is a superset of (instead of equivalent to) the value spaces of primitive base datatypes and plain literals; hence, typed literals with unsupported predicates are interpreted more intuitively.

\section{Related work}

The concrete domain approach [2,14] provides a rigorous treatment of datatype predicates, rather than datatypes. ${ }^{12}$ In the type system approach [11], datatypes are considered to be sufficiently structured by type systems; however, it does not specify how the derivation mechanism of a type system affects the set of datatypes D. An early version of [5] suggests some solutions to the problem of referring to an XML Schema user defined simple type with a URI reference; however, it does not address the computability issue of combining the $\mathcal{S H O I N D L}$ with customised datatypes. The current version of this $\mathrm{W} 3 \mathrm{C}$ technical report refers to our work on unary datatype groups, as a solution to the problem of combining OWL DL with customised datatypes. It is worth mentioning that the SPARQL query language for RDF [20] allows the use not only of datatypes, but also of some datatype predicates and operators defined in [15]. SPARQL does not, however, allow the use of customised datatypes or datatype predicates. Furthermore, the eq operator SPARQL supports is not an equivalence relation because of some so-called "corner cases" [5].

\section{Conclusion}

Although OWL is rather expressive, it has a very serious limitation on datatypes; i.e., it does not support customised datatypes. It has been pointed out that many potential users will not adopt OWL unless this limitation is overcome. Accordingly, the Semantic Web Best Practices and Development Working Group has set up a task force to address this issue. As discussed above, a solution to the problem should cover much more than just a standard way of referring to an XML Schema user defined simple type with a URI reference.

In this paper, we propose OWL-Eu, an extension of OWL DL that supports customised datatypes. The underpinning of OWLEu is the $\mathcal{S H O I N}\left(\mathcal{G}_{1}\right)$ DL, a combination of $\mathcal{S H O I N}$ and a unary datatype group. OWL-Eu is decidable if the combined unary datatype group is conforming; conformance of a unary datatype group precisely specifies the conditions on the set of supported datatypes. OWL-Eu provides a general framework for integrating OWL DL with customised datatypes, such as XML Schema non-list simple types.

\footnotetext{
12 The reader is referred to Section 5.1 .3 of [16] for detailed discussions on concrete domains.
} 
We have implemented a prototype extension of the FaCT [9] DL system, called FaCt-DG, to support TBox reasoning in the $\mathcal{S H \mathcal { I }}\left(\mathcal{G}_{1}\right)$ DL, a sub-language of OWL-Eu. As for future work, we are planing to extend the DIG1.1 interface [7] to support OWL-Eu, and to implement a protégé [13] plug-in to support XML Schema non-list simple types, i.e. users should be able to define and/or import customised XML Schema non-list simple types based on a set of supported datatypes, and to exploit our prototype through the extended DIG interface.

\section{References}

[1] H. Alvestrand, Rfc 3066-tags for the identification of languages, Technical report, IETF, January 2001, http://www.isi.edu/in-notes/rfc3066.txt.

[2] F. Baader, P. Hanschke, A Schema for integrating concrete domains into concept languages, Proceedings of the 12th International Joint Conference on Artificial Intelligence (IJCAI'91), 1991, pp. 452-457.

[3] S. Bechhofer, F. van Harmelen, J. Hendler, I. Horrocks, D.L. McGuinness, P.F. Patel-Schneider, L.A. Stein (Eds.), OWL Web Ontology Language Reference, http://www.w3.org/TR/owl-ref/, February 2004.

[4] P.V. Biron, A. Malhotra, Extensible Markup Language (XML) Schema Part 2: Datatypes - W3C Recommendation 02 May 2001, Technical report, World Wide Web Consortium, 2001, http://www.w3.org/TR/xmlschema$2 /$.

[5] J.J. Carroll, J.Z. Pan, XML Schema Datatypes in RDF and OWL, Technical report, W3C Semantic Web Best Practices and Development Group, November 2004, Editors' Draft, http://www.w3.org/2001/ sw/BestPractices/XSCH/xsch-sw/.

[6] Unicode Consortium, The Unicode Standard, Addison-Wesley, 2000, ISBN 0-201-61633-5, version 3.

[7] DIG, SourceForge DIG Interface Project, http://sourceforge.net/projects/ dig/, 2004

[8] P. Hayes, RDF semantics, Technical report, W3C, February 2004, W3C recommendation, http://www.w3.org/TR/rdf-mt/.

[9] I. Horrocks, Using an expressive description logic: fact or fiction? Proceedings of the KR'98, 1998, pp. 636-647.

[10] I. Horrocks, P.F. Patel-Schneider, F. van Harmelen, From SHIQ and RDF to OWL: the making of a web ontology language, J. Web Semantics 1 (1) (2003) 7-26.
[11] I. Horrocks, U. Sattler, Ontology reasoning in the $\mathcal{S H O Q}(D)$ description logic, Proceedings of the 17th International Joint Conference on Artificial Intelligence (IJCAI 2001), 2001, pp. 199-204.

[12] I. Horrocks, U. Sattler, S. Tobies, Practical reasoning for expressive description logics, Proceedings of the International Conference on Logic for Programming and Automated Reasoning (LPAR'99), 1999 number 1705 in LNAI, pp. 161-180.

[13] H. Knublauch, R.W. Fergerson, N.F. Noy, M.A. Musen, The Protégé OWL Plugin: An Open Development Environment for Semantic Web Applications, International Semantic Web Conference, 2004, pp. 229-243.

[14] C. Lutz, The complexity of reasoning with concrete domains, Ph.D. thesis, Teaching and Research Area for Theoretical Computer Science, RWTH Aachen, 2001

[15] A. Malhotra, J. Melton, N. Walsh, XQuery 1.0 and XPath 2.0 Functions and Operators, W3C Working Draft, http://www.w3.org/TR/xpath-functions, July 2004

[16] J.Z. Pan, Description logics: reasoning support for the semantic web, Ph.D. thesis, School of Computer Science, The University of Manchester, Oxford Road, Manchester M13 9PL, UK, 2004.

[17] J.Z. Pan, I. Horrocks, Extending datatype support in web ontology reasoning, Proceedings of the 2002 International Conference on Ontologies, Databases and Applications of SEmantics (ODBASE 2002), October 2002.

[18] J.Z. Pan, I. Horrocks, Web ontology reasoning with datatype groups, Proceedings of the 2003 International Semantic Web Conference (ISWC2003), 2003, pp. 47-63.

[19] P.F. Patel-Schneider, P. Hayes, I. Horrocks, OWL web ontology language semantics and abstract syntax, Technical report, W3C, February 2004, W3C Recommendation, http://www.w3.org/TR/2004/REC-owlsemantics-20040210.

[20] E. Prud'hommeaux, A. Seaborne (Eds.), SPARQL Query Language for RDF, W3C Working Draft, http://www.w3.org/TR/2004/WD-rdfsparql-query-20041012, December 2004.

[21] RDF-Logic Mailing List, http://lists.w3.org/archives/public/www-rdflogic/. W3C Mailing List, starts from 2001.

[22] A. Rector, Re: [UNITS, OEP] FAQ: Constraints on data values range, Discussion in [23], April 2004, http://lists.w3.org/Archives/ Public/public-swbp-wg/2004Apr/0216.html.

[23] Semantic Web Best Practice and Development Working Group Mailing List, http://lists.w3.org/archives/public/public-swbp-wg/, W3C Mailing List, starts from 2004.

[24] S. Tobies, Complexity results and practical algorithms for logics in knowledge representation, Ph.D. thesis, Rheinisch-Westf'alischen Technischen Hochschule Aachen, 2001, http://lat.inf.tu-dresden.de/ research/phd/Tobies-PhD-2001.pdf. 